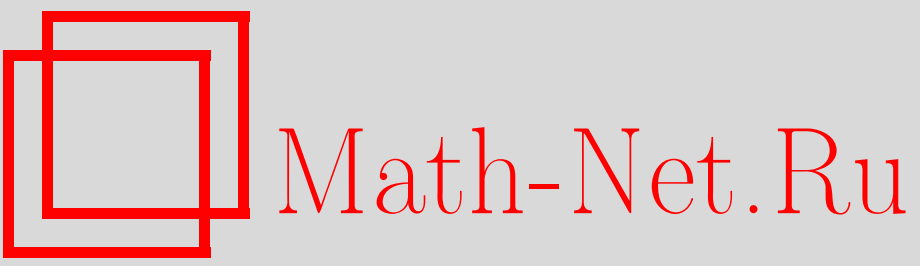

И. В. Колесникова, Трехмодовые прогибы сжатой балки на двойном упругом основании в модифицированной модели Власова-Леонтьева, Итоги науки и техн. Сер. Соврем. мат. и ее прил. Темат. обз., 2021, том 190, 115-121

DOI: https://doi.org/10.36535/0233-6723-2021-190-115-121

Использование Общероссийского математического портала Math-Net.Ru подразумевает, что вы прочитали и согласны с пользовательским соглашением

http: //www. mathnet.ru/rus/agreement

Параметры загрузки:

IP : 3.81 .55 .215

26 апреля 2023 г., 18:18:59 


\title{
ТРЕХМОДОВЫЕ ПРОГИБЫ СЖАТОЙ БАЛКИ
} НА ДВОЙНОМ УПРУГОМ ОСНОВАНИИ В МОДИФИЦИРОВАННОЙ МОДЕЛИ ВЛАСОВА-ЛЕОНТЬЕВА

\author{
(c) 2021 г. $\quad$ И. В. КОЛЕСНИКОВА
}

\begin{abstract}
АннотАция. В работе представлен алгоритм приближенного вычисления и анализа закритических прогибов продольно сжатой упругой балки на двойном упругом основании в модифицированной модели Власова-Леонтьева. Процедура основана на применении вариационного метода Пуанкаре-Ляпунова-Шмидта, позволяющего сводить анализ закритических деформаций балки к анализу критических точек ключевой функции на конечномерном пространстве ключевых переменных. Метод Ляпунова-Шмидта позволяет вычислять закритические прогибы балки, определять устойчивость бифурцирующих состояний и анализировать строение каустики (дискриминантного множества) в пространстве управляющих параметров. Основная идея заключена в сведении (редукции) задачи изучения изгибов балки к дискриминантному анализу ветвления критических точек полинома от трех переменных (главной части ключевой функции ЛяпуноваШмидта).
\end{abstract}

Ключевые слова: модель Власова-Леонтьева, обобщенные краевые условия Дирихле, функционал энергии, мода прогиба, ключевая функция Ляпунова-Шмидта, ветвь прогиба, каустика.

\section{THREE-MODE BENDINGS OF A COMPRESSED BEAM ON A DOUBLE ELASTIC FOUNDATION IN THE MODIFIED VLASOV-LEONTYEV MODEL}

\section{(c) 2021 I. V. KOLESNIKOVA}

\begin{abstract}
In this paper, we present an algorithm for approximate calculation and analysis of supercritical bendings of a longitudinally compressed, elastic beam on a double elastic foundation in the modified Vlasov-Leontiev model. The procedure is based on the Poincaré-Lyapunov-Schmidt variational method, which allows one to reduce the analysis of supercritical deformations of a beam to the analysis of critical points of the key function on the finite-dimensional space of key variables. The Lyapunov-Schmidt method allows one to calculate supercritical bendings of the beam, to determine the stability of bifurcating states, and to analyze the structure of the caustic (discriminant set) in the space of control parameters. The basic idea is the reduction of the problem on bendings of a beam to the discriminant analysis of branching of critical points of a polynomial in three variables (the principal part of the Lyapunov-Schmidt key function).
\end{abstract}

Keywords and phrases: Vlasov-Leontyev model, generalized Dirichlet boundary conditions, energy functional, bending mode, Lyapunov-Schmidt key function, branch of bending, caustic.

AMS Subject Classification: 35L05 
1. Введение. Изначально модифицированная модель Власова-Леонтьева была предложена в [3], где она представлена в виде обыкновенного дифференциального уравнения шестого порядка

$$
-\frac{d^{6} w}{d x^{6}}+a_{3} \frac{d^{4} w}{d x^{4}}-a_{2} \frac{d^{2} w}{d x^{2}}+a_{1} w=p
$$

при обобщенных краевых условиях Дирихле

$$
\frac{d^{4} w}{d x^{4}}(0)=\frac{d^{4} w}{d x^{4}}(1)=\frac{d^{2} w}{d x^{2}}(0)=\frac{d^{2} w}{d x^{2}}(1)=w(1)=w(0)=0,
$$

$w=w(x)$ - функция прогиба средней линии балки, $a_{1}, a_{2}, a_{3}$-положительные механические константы, $p=p(x)$ - функциональный параметр внешней нагрузки, $x-$ (промасштабированный) параметр длины средей линии балки. Уравнение (1) имеет единственное рещение в пространстве $H^{6}$ соболевских функций класса $W_{2}^{6}[0,1]$, удовлетворяющих краевым условиям $(2)$, что объясняется положительной определенностью симметричного оператора

$$
-\frac{d^{6} w}{d x^{6}}+a_{3} \frac{d^{4} w}{d x^{4}}-a_{2} \frac{d^{2} w}{d x^{2}}+a_{1} w
$$

(при выполнении краевых условий (2)).

Уравнение (1) является обобщением известного уравнения Фусса-Винклера-Циммермана

$$
\frac{d^{4} w}{d x^{4}}-a_{2} \frac{d^{2} w}{d x^{2}}+a_{1} w=p
$$

из строительной механики (см. [3]). Рассмотренная ниже модификация предполагает наличие продольного сжатия балки, приводящего к смене знаков перед коэффициентами в модельном уравнении, что дает эффект трехмодового вырождения в нулевом состоянии:

$$
\frac{d^{6} w}{d x^{6}}+a_{3} \frac{d^{4} w}{d x^{4}}+a_{2} \frac{d^{2} w}{d x^{2}}+a_{1} w-w^{3}=p .
$$

Наличие нелинейного слагаемого позволяет «контролировать» рост амплитуд посткритических прогибов балки (см. [1]).

Основой примененной ниже методики исследования закритических прогибов служит вариационная версия метода Ляпунова-Шмидта (см. $[7,11,15])$, позволяющая не только вычислять прогибы, но и определять их устойчивость, проводить построение каустики (дискриминантного множества) в пространстве управляющих параметров $a=\left(a_{1}, a_{2}, a_{3}\right)$. Центральная конструктивная идея - сведе́ние (редукция) задачи об изучении бифуркации изгибов к анализу ветвления критических точек полинома от шести переменных, являющегося главной частью ключевой функции

$$
W(\xi, \delta, q)=\inf _{w:\left\langle w, e_{k}\right\rangle=\xi_{k}} V(w, a, q)=V\left(\sum_{i=1}^{3} \xi_{i} e_{i}+\Phi(\xi)\right)
$$

где

$$
V(\xi, \delta, q):=\int_{0}^{1}\left(\frac{1}{2}\left(\left(\frac{d^{3} w}{d x^{3}}\right)^{2}-a_{3}\left(\frac{d^{2} w}{d x^{2}}\right)^{2}+a_{2}\left(\frac{d w}{d x}\right)^{2}-a_{1} w^{2}\right)+\frac{1}{4} w^{4}+p w\right)
$$

- потенциал уравнения (3), $e_{1}, e_{2}, e_{3}$ - моды прогиба в нуле при соответствующем значении векторного параметра $a=\bar{a}, \delta:=a-\bar{a}$. Уравнение (3) является уравнением Эйлера для экстремалей функционала (5).

2. Построение ключевой функции при $p=0$. Колебания и волновые движения упругой балки на упругом основании изучали Ю. А. Митропольский и Б. И. Мосеенков (см. [12]), J. M. Т. Thompson и G. W. Hunt (см. [16,17]), Б. С. Бардин и С. Д. Фурта (см. [2]) и др.

Обобщенная нелинейная (промасштабированная) модель закритических прогибов однородной балки на двойном упругом основании представлена уравнением (3), в котором $w=w(x)$ - прогиб балки (поле смещений точек средней линии упругой балки, расположенной вдоль оси $x$ ). 
Изучение закритических прогибов состоит в отыскании решений краевой задачи (3)-(2) при различных значениях параметров. Данная краевая задача может допускать трехмерное вырождение, порождающее трехмодовые бифуркации с разнообразными геометрическими и физическими эффектами.

Решение этой задачи будем осуществлять посредством вариационной версии метода конечномерной редукции Ляпунова-Шмидта (см. [7,11,15], сводящей анализ краевой задачи к описанию ветвления экстремалей параметрического семейства полиномов от трех переменных - главной части ключевой функции (4).

Будем далее рассматривать пример, в котором тройка мод бифуркации задана набором функций

$$
e_{1}=\sqrt{2} \sin (\pi x), \quad e_{2}=\sqrt{2} \sin (2 \pi x), \quad e_{3}=\sqrt{2} \sin (3 \pi x)-
$$

базисом ядра второго дифференциала, соответствующим (критическому) значению управляющего параметра

$$
a=\bar{a}:=\left(36 \pi^{6}, 49 \pi^{4}, 14 \pi^{2}\right) .
$$

После пересечения точкой $a=\left(a_{1}, a_{2}, a_{3}\right)$ произвольной характеристической плоскости (в $\left.\mathbb{R}^{3}\right)$ нулевая функция теряет стабильность и вблизи нее рождаются ненулевые стабильные состояния. Характеристические плоскости в общем случае задаются посредством линеаризованного уравнения

$$
\frac{d^{6} w}{d x^{6}}+a_{3} \frac{d^{4} w}{d x^{4}}+a_{2} \frac{d^{2} w}{d x^{2}}+a_{1} w=0
$$

(при краевых условиях (2)). Характеристические плоскости состоят из тех и только тех точек $a$, при которых уравнение (6) имеет ненулевое решение. Поиск нетривиальных решений линеаризованного уравнения осуществляется через характеристическое уравнение

$$
-\lambda^{6}+a_{3} \lambda^{4}-a_{2} \lambda^{2}+a_{1}=0 .
$$

Учет краевых условий с необходимостью приводит к соотношениям $\lambda^{2}=n^{2} \pi^{2}, n=1,2, \ldots$, из которого получаем набор равенств:

$$
a_{1}-a_{2} n^{2} \pi^{2}+a_{3} n^{4} \pi^{4}-n^{6} \pi^{6}=0,
$$

задающих характеристические плоскости $L_{1}, L_{2}, \ldots, L_{n}, \ldots$ Поверхность $L$, огибающая семейства характеристических плоскостей, выделяет область в $\mathbb{R}^{3}$, состоящей из точек, для которых соответствующий функционал действия имеет в нуле единственную точку минимума. При пересечении точкой $a$ поверхности $L\left(\right.$ в $\left.\mathbb{R}^{3}\right)$ происходит бифуркация рождения нетривиальных стабильных состояний. Поверхность $L$ является кусочно линейной, гранями которой служат плоские многоугольники, ограниченные линиями попарных пересечений характеристических плоскостей. Эти линии также будем называть характеристическими. Общие точки для отдельных пар характеристических линий будем называть характеристическими точками. Интерес к последним вызван тем, что они дают эффект трехмодового вырождения. Точки характеристических линий, отличные от характеристических точек, дают эффект двухмодового вырождения. Пересечение поверхности $L$ по внутренней точке грани, принадлежащей $L_{n}$, приводит к одномерной бифуркации с модой $e_{n}=\sqrt{2} \sin (\pi n x)$. Пересечение $L$ по точке излома, принадлежащей линии (характеристической) пересечения плоскостей $L_{n}, L_{m}$ приводит к двумерной бифуркации с модами $e_{n}, e_{m}$. Соответственно, пересечение $L$ по характеристической точке, являющейся точкой пересечения плоскостей $L_{n}, L_{m}, L_{l}$, приводит к трехмерной бифуркации с модами $e_{n}, e_{m}, e_{l}$. Три характеристические плоскости $L_{m}, L_{n}, L_{l}$, отвечающие произвольной тройке попарно различных натуральных чисел $m, n, l$, пересекаются по единственной точке

$$
\left(a_{1}, a_{2}, a_{3}\right)=\left(n^{2} m^{2} l^{2} \pi^{6},\left(n^{2} m^{2}+m^{2} l^{2}+n^{2} l^{2}\right) \pi^{4},\left(n^{2}+m^{2}+l^{2}\right) \pi^{2}\right) .
$$


Доказательство этого факта вытекает из того, что характеристическая точка определяется системой уравнений

$$
\left\{\begin{array}{l}
a_{1}-n^{2} \pi^{2} a_{1}+n^{4} \pi^{4} a_{3}-n^{6} \pi^{6}=0, \\
a_{1}-m^{2} \pi^{2} a_{2}+m^{4} \pi^{4} a_{3}-m^{6} \pi^{6}=0, \\
a_{1}-l^{2} \pi^{2} a_{2}+l^{4} \pi^{4} a_{3}-l^{6} \pi^{6}=0 .
\end{array}\right.
$$

Здесь $l, m, n$ - натуральные попарно различные числа. Этой системе уравнений отвечает единственное решение (7).

В частном случае $l=1, m=2, n=3$, получаем ранее выбранный пример. В случае других наборов мод исследования проводятся аналогичным образом.

3. Построение главной части ключевой функции в случае $3 D$-вырождения. Анализ бифуркационных эффектов, происходящих при локализации управляющих параметров вблизи точки $\bar{a}=(1,2,3): a_{k}=\bar{a}_{k}+\delta_{k}, k=1,2,3$, можно осуществить посредством вариационной версии редукции Ляпунова-Шмидта (см. [7]) к анализу ключевой функции (4) от трех ключевых переменных, которую запишем в виде

$$
W(\xi, \delta)=\inf _{w:\left\langle w, e_{k}\right\rangle=\xi_{k}, k=1,2,3} V(w, \bar{a}+\delta),
$$

где $\xi=\left(\xi_{1}, \xi_{2}, \xi_{3}\right)^{\top}, \delta=\left(\delta_{1}, \delta_{2}, \delta_{3}\right)^{\top}$. Известно, что в рассматриваемых условиях функция $W(\xi, \delta)$ является гладкой. Эта функция наследует все аналитические и топологические свойства $V$ на $E$ (см. [7]). В частности, при достаточно малых $\delta$ существует взаимно однозначное соответствие между критическими точками $V$ и $W$, сохраняющее типы точек (кратности, значения индекса Морса и т. пг.).

Так как функционал $V$ четен, т.е. $V(-w, a)=V(w, a)$, и симметричен относительно инволюции $I_{1}: w(x) \mapsto w(1-x)$, то $V$ инвариантен относительно действия группы $\mathbb{Z}_{2}^{2}$, заданного парой инволюций $I_{1}, I_{2}$, где $I_{2}=-I_{1}$. Очевидно, что выполняются соотношения

$$
I_{1}\left(e_{1}\right)=e_{1}, \quad I_{1}\left(e_{2}\right)=-e_{2}, \quad I_{1}\left(e_{3}\right)=e_{3} .
$$

Следовательно,

$$
W\left(-\xi_{1}, \xi_{2},-\xi_{3}, \delta_{1}, \delta_{2}, \delta_{3}\right)=W\left(\xi_{1},-\xi_{2}, \xi_{3}, \delta_{1}, \delta_{2}, \delta_{3}\right)=W\left(\xi_{1}, \xi_{2}, \xi_{3}, \delta_{1}, \delta_{2}, \delta_{3}\right) .
$$

Учитывая эту симметрию, можно записать следующее асимптотическое представление ключевой функции:

$$
W(\xi, \delta)=U(\xi, \delta)+o\left(|\xi|^{4}\right)+O\left(|\xi|^{4}\right) O(\delta),
$$

где

$$
U(\xi, \delta)=V\left(\xi_{1} e_{1}+\xi_{2} e_{2}+\xi_{3} e_{3}, \delta\right)
$$

- ритцевская аппроксимация функционала $V$ по модам $e_{1}, e_{2}, e_{3}$. Непосредственные вычисления приводят к следующему утверждению.

Теорема 1. Для ключевой функиии (8) имеет место асимптотическое представление

$$
\begin{aligned}
W(\xi, \delta)=\frac{1}{2}\left(\bar{\delta}_{1} \xi_{1}^{2}+\bar{\delta}_{2} \xi_{2}^{2}+\bar{\delta}_{3} \xi_{3}^{2}\right) & +\frac{3}{8}\left(\xi_{1}^{4}+\xi_{2}^{4}+\xi_{3}^{4}\right)+ \\
+ & \frac{3}{2}\left(\xi_{1}^{2} \xi_{2}^{2}+\xi_{1}^{2} \xi_{3}^{2}+\xi_{2}^{2} \xi_{3}^{2}+\xi_{1} \xi_{2}^{2} \xi_{3}-\frac{1}{3} \xi_{1}^{3} \xi_{3}\right)+o\left(|\xi|^{4}\right)+O\left(|\xi|^{4}\right) O(\delta),
\end{aligned}
$$

где

$$
\bar{\delta}_{1}=\left(-\delta_{1}+\delta_{2}-\delta_{3}\right) \pi^{2}, \quad \bar{\delta}_{2}=\left(-\delta_{1}+4 \delta_{2}-16 \delta_{3}\right) \pi^{4}, \quad \bar{\delta}_{3}=\left(-\delta_{1}+9 \delta_{2}-81 \delta_{3}\right) \pi^{6} .
$$

Доказательство. Доказательство вытекает из следующих соотношений: 


$$
\begin{aligned}
& W(\xi, \delta)= V\left(\xi_{1} e_{1}+\xi_{2} e_{2}+\xi_{3} e_{3}, \delta\right)=\int_{0}^{1} \frac{1}{2}\left(\left(-\left(\frac{d^{3}\left(\xi_{1} e_{1}+\xi_{2} e_{2}+\xi_{3} e_{3}\right)}{d x^{3}}\right)^{2}+\right.\right. \\
&+\left(14+\delta_{3}\right)\left(\frac{d^{2}\left(\xi_{1} e_{1}+\xi_{2} e_{2}+\xi_{3} e_{3}\right)}{d x^{2}}\right)^{2}-\left(49+\delta_{2}\right)\left(\frac{d\left(\xi_{1} e_{1}+\xi_{2} e_{2}+\xi_{3} e_{3}\right)}{d x}\right)^{2}+ \\
&\left.\left.+\left(36+\delta_{1}\right)\left(\xi_{1} e_{1}+\xi_{2} e_{2}+\xi_{3} e_{3}\right)^{2}+\frac{\left(\xi_{1} e_{1}+\xi_{2} e_{2}+\xi_{3} e_{3}\right)^{4}}{4}\right)\right) d x \\
& \int_{0}^{1}\left(\frac{d^{3}\left(\xi_{1} e_{1}+\xi_{2} e_{2}+\xi_{3} e_{3}\right)}{d x^{3}}\right)^{2}=\left(\xi_{1}^{2}+64 \xi_{2}^{2}+729 \xi_{3}^{2}\right) \pi^{6} \\
& \int_{0}^{1}\left(\frac{d^{2}\left(\xi_{1} e_{1}+\xi_{2} e_{2}+\xi_{3} e_{3}\right)}{d x^{2}}\right)^{2}=\left(\xi_{1}^{2}+16 \xi_{2}^{2}+81 \xi_{3}^{2}\right) \pi^{4} \\
& \int_{0}^{1}\left(\frac{d\left(\xi_{1} e_{1}+\xi_{2} e_{2}+\xi_{3} e_{3}\right)}{d x}\right)^{2}=\left(\xi_{1}^{2}+4 \xi_{2}^{2}+9 \xi_{3}^{2}\right) \pi^{2} \\
& \int_{0}^{1}\left(\xi_{1} e_{1}+\xi_{2} e_{2}+\xi_{3} e_{3}\right)^{4} d x=\frac{3}{2}\left(\xi_{1}^{4}+\xi_{2}^{4}+\xi_{3}^{4}\right)+6\left(\xi_{1}^{2} \xi_{2}^{2}+\xi_{1}^{2} \xi_{3}^{2}+\xi_{2}^{2} \xi_{3}^{2}+\xi_{1} \xi_{2}^{2} \xi_{3}\right)-2 \xi_{1}^{3} \xi_{3} . \\
& \int_{0}^{1}\left(\xi_{1} e_{1}+\xi_{2} e_{2}+\xi_{3} e_{3}\right)^{2} d x=\xi_{1}^{2}+\xi_{2}^{2}+\xi_{3}^{2},
\end{aligned}
$$

Подставив полученные значения для интегралов в $W(\xi, \delta)$, получим искомое выражение.

Замечание 1. «Геометрический сюжет» бифуркации критических точек и первые асимптотики ветвей бифурцирующих точек (по закритическим приращениям управляющих параметров) для функции $W(\xi, \delta)$ полностью определяются ее главной частью $U(\xi, \delta)$ (см. [7]).

Замечание 2. В случае ненулевого, но малого функционального параметра $p$ изложенная схема немного усложняется за счет появления в уравнениях и аналитических выкладках дополнительных слагаемых. Можно ограничиться рассмотрением разложения по основным модам: $p(x)=\eta_{1} e_{1}+\eta_{2} e_{2}+\eta_{2} e_{3}$. Членами разложения Фурье по остальным модам можно пренебречь, так как они вносят малый вклад в окончательное асимптотическое представление решений (по сравнению с параметрами $\left.\delta_{j}, \eta_{k}\right)$.

4. Анализ ключевой функции посредством вторичной редукции (к функции двух переменных). Так как функция (10) обладает симметрией относительно группы $\mathbb{Z}_{2}^{2}$, заданного парой инволюций $I_{1}, I_{2}$, где $I_{2}=-I_{1}, I_{1}: w(x) \mapsto w(1-x)$, то эта функция четна по переменной $\xi_{2}: U\left(\xi_{1}, \xi_{2}, \xi_{3}, \delta\right)=U\left(\xi_{1},-\xi_{2}, \xi_{3}, \delta\right)$. Следовательно, она может быть представлена в виде

$$
U(\xi, \delta)=\frac{3}{8} \xi_{2}^{4}+\frac{p}{2} \xi_{2}^{2}+q
$$

где

$$
p=\bar{\delta}_{2}+3\left(\xi_{1}^{2}+\xi_{1}^{2}+\xi_{1} \xi_{3}\right), \quad q=\frac{1}{2}\left(\bar{\delta}_{1} \xi_{1}^{2}+\bar{\delta}_{3} \xi_{3}^{2}\right)+\frac{3}{8}\left(\xi_{1}^{4}+\xi_{3}^{4}+4 \xi_{1}^{2} \xi_{3}^{2}\right)-\frac{1}{2} \xi_{1}^{3} \xi_{3} .
$$

Такое представление функции $U$ дает возможность вторичной редукции: дальнейшего сокращения числа переменных до двух (редукции к функции двух переменных). При этом выделяются два случая: $p \geqslant 0$ и $p \leqslant 0$. При $\bar{\delta}_{2} \leqslant 0$ эти соотношения выделяют в плоскости переменных $\xi_{1}$, $\xi_{3}$ внешность и внутренность эллипса $\bar{\delta}_{2}+3\left(\xi_{1}^{2}+\xi_{1}^{2}+\xi_{1} \xi_{3}\right)=0$. При $\bar{\delta}_{2} \geqslant 0$ неравенство $p \geqslant 0$ выполняется для всех значений $\xi_{1}, \xi_{3}$. 
В случае $p \geqslant 0$ можно рассмотреть вторично редуцированную ключевую функцию

$$
q=q\left(\xi_{1}, \xi_{3}\right):=\inf _{\xi_{2}} U
$$

В предполагаемых условиях функция $q$ наследует (вблизи нуля) аналитические и топологические свойства функции $U(\xi)$. Причем имеется естественное взаимно однозначное соответствие $\left(\xi_{1}, \xi_{3}\right) \mapsto\left(\xi_{1}, 0, \xi_{3}\right)$ между критическими точками функций $q$ и $U$, сохраняющее топологические типы точек.

В случае $p \leqslant 0$ получаем (вторично) редуцированную ключевую функцию $\inf _{\xi_{2}} U$ двух видов:

(1) $U_{1}=U\left(\xi_{1}, 0, \xi_{3}\right)=q$; при этом соответствие $\left(\xi_{1}, \xi_{3}\right) \mapsto\left(\xi_{1}, 0, \xi_{3}\right)$ (между критическими точками функций $q$ и $U)$ повышает индекс Морса на единицу;

(2) $U_{2}=U\left(\xi_{1}, \pm \sqrt{-2 p / 3}, \xi_{3}\right)$; при этом соответствие $\left(\xi_{1}, \xi_{3}\right) \mapsto\left(\xi_{1}, \pm \sqrt{-2 p / 3}, \xi_{3}\right)$ (между критическими точками функций $U_{1}$ и $U$ ) сохраняет индекс Морса.

Таким образом, вторичная редукция сводит изучение критических точек к описанию каустик и раскладов бифурцирующих экстремалей для функций с особенностями двумерных сборок. Достаточно полный анализ таких функций проведен в работах $[9,10]$.

Исследование параметрических разверток двумерных сборок проводится по единой схеме: после приведения к нормальной форме и перехода к полярным координатам редуцированная ключевая функция приобретает удобный для исследования каустики и $b i f$-раскладов (см. $[4,5,7,11,15])$, причем эти исследования можно провести посредством элементарных методов математического анализа.

\section{СПИСОК ЛИТЕРАТУРЫ}

1. Арнольд В. И. «Жёсткие» и «мягкие» математические модели. - М.: МЦНМО, 2008.

2. Бардин Б. С., Фурта С. Д. Локальная теория существования периодических волновых движений бесконечной балки на нелинейно упругом основании// в кн.: Актуальные проблемы классической и небесной механики. - М.: Эльф, 1998. - С. 13-22.

3. Власов В. З., Леонтъев Н. Н. Балки, плиты и оболочки на упругом основании. - М.: Физматгиз, 1960.

4. Гнеушев И. А., Колесникова И. В., Костин Д. В., Сапронов Ю. И. Прогибы сжатой балки на двойном упругом основании (в обобщенной модели Власова-Леонтьева)// Вестн. Воронеж. ун-та. Сер. Физ. Мат. - 2018. - 2. - С. 173-181.

5. Даринский Б. М., Колесникова И. В., Сапронов Ю. И. Ветвление сегнетоэлектрических фаз неоднородного кристалла вблизи критической фазы с трехмерной особенностью шестого порядка// Вестн. Воронеж. ун-та. Сер. Физ. Мат. - 2009. - 3. - С. 101-107.

6. Даринский Б. М., Сапронов Ю. И. Бифуркации экстремалей вблизи особенности многомерной сборки// Изв. вузов. Мат. - 1997. - № 2. - С. 35-46.

7. Даринский Б. М., Сапронов Ю. И., Царев С. Л. Бифуркации экстремалей фредгольмовых функционалов// Совр. мат. Фундам. напр. - 2004. - 12. - С. 3-140.

8. Зачепа А. В., Сапронов Ю. И. О бифуркации экстремалей фредгольмова функционала из вырожденной точки минимума с особенностью 3-мерной сборки// Тр. мат. фак-та Воронеж. ун-та. - 2005. 9. - C. $57-71$.

9. Костин Д. В. Применение формулы Маслова для асимптотического решения одной задачи об упругих деформациях// Мат. заметки. - 2008. - 83, № 1. - С. 50-60.

10. Костин Д. В. Об одной схеме анализа двухмодовых прогибов слабо неоднородной упругой балки// Докл. РАН. - 2008. - 418, № 4. - С. 295-299.

11. Костин Д. В., Сапронов Ю. И. Функциональный анализ и многомодовые прогибы упругих систем. - Воронеж: ИПЦ ВГУ, 2012.

12. Мітропольский Ю. О., Мосеенков Б. І. Дослідження коливань в системах з розподіленими параметрами (асимптотичні методи). - Київ, 1961.

13. Сапронов Ю. И. Многомодовые бифуркации упругих равновесий// Прикл. мат. мех. - 1988. - 52, № 6. - С. 997-1006.

14. Сапронов Ю. И. Полурегулярные угловые особенности гладких функций// Мат. сб. - 1989. - 180, № 10. - С. 1299-1310. 
15. Сапронов Ю. И. Конечномерные редукции в гладких экстремальных задачах// Усп. мат. наук. - 1996. - 51, № 1. - C. 101-132.

16. Thompson J. M. T. Advances in shell buckling : Theory and experiments// Int. J. Bifurcat. Chaos. — 2015. - 25, № 1. - P. 1-25.

17. Thompson J. M. T., Hunt G. W. A General Theory of Elastic Stability. — Wiley, 1973.

Колесникова Инна Викторовна

Воронежский государственный университет

E-mail: kolinna@inbox.ru 